\section{SIMSTAT: Bootstrap computer simulation and statistical program for IBM personal computers}

\author{
NORMAND PÉLADEAU \\ Unité de Recherche Psychosociale \\ Centre de Recherche de l'Hôpital Douglas \\ Verdun, Quebec, Canada \\ and \\ YVES LACOUTURE \\ École de Psychologie, Université Laval \\ Laval, Quebec, Canada
}

\begin{abstract}
SIMSTAT is a computer program that provides bootstrap confidence intervals and estimates distribution parameters for 27 univariate and bivariate statistics. Because it permits variation of the bootstrap sample size, it is a convenient tool for research planning, allowing comparison of power estimates and estimator precision for various estimators and/or sample sizes. SIMSTAT also is a useful tool for studying the robustness of various statistics on real-world data and may be helpful in teaching statistical principles.
\end{abstract}

Most parametric statistical procedures used in behavioral sciences share the same basic assumptions and postulate normal data distribution in the population. As demonstrated by Micceri (1989), most measures commonly used in the behavioral sciences are not distributed according to a normal probability distribution. Moreover, Micceri argues that research on statistical robustness has seldom involved distributions matching those encountered in social or behavioral sciences. This raises doubts about the relevance of such studies and highlights the need for further research involving real-world data or more realistic data distributions. When parametric methods are inadequate, the social scientist often relies on nonparametric procedures for data analysis. Unfortunately, these procedures still require basic assumptions regarding data distribution and, moreover, they are often associated with loss of information. In recent years, a resampling technique known as bootstrap simulation has been developed that may offer a compromise solution. Proposed by Efron (Diaconis \& Efron, 1983; Efron, 1981), this technique relaxes some assumptions about the distribution of the estimators through nonparametric estimation of sampling distributions. ${ }^{1}$

The idea underlying the bootstrap is quite simple: the sample data are treated as though they constitute the data

Address correspondence to N. Péladeau, Unité de Recherche Psychosociale, Hôpital Douglas, 6875 Lasalle Blvd., Verdun QC, Canada H4H 1 R3. from the entire population. The sample data are used as a pool from which a large number of samples-each the size of the original sample-are drawn with replacement. For each resampling, the value of the estimator (e.g., correlation coefficient) is computed. The resulting bootstrap estimators are used to estimate the sampling variability. It has been demonstrated that this approach provides a good approximation of the distribution of the estimators (Diaconis \& Efron, 1983; Efron, 1981; Lunnenborg, 1987). Therefore, it becomes possible to describe some of the sampling properties of an estimator, such as its standard error, and compute confidence intervals.

We can illustrate this process by replicating the "law school experiment," presented by Diaconis and Efron (1983). These authors tried to estimate the sampling distribution of the correlation between the average undergraduate grade point average and the average score on the law school admission test from a sample of 15 law schools. The data obtained (see Appendix A) yielded a correlation coefficient of .776. To estimate the accuracy of this statistic, the program randomly selects 15 bivariate observations from the original sample, with replacement, and computes a correlation coefficient for this new sample. In a given sample, some schools may be selected more than once whereas others may not be included, thereby producing a different correlation coefficient. Repeating this sampling process a number of times provides information about the distribution of the correlation estimator for that specific sample, as if the distribution were constructed from real samples. An example output for a bootstrap simulation analysis consisting of 5,000 samples is listed in Appendix B, which shows that $68 \%$ of the samples yielded a correlation coefficient between .6376 and .9068 , and $90 \%$ of the correlation coefficients are between .5217 and .9470 . In addition to the confidence intervals, the output includes a graph of the distribution, a percentile table, and various descriptive statistics for the estimated distribution.

The main advantage of the bootstrap approach is that the distribution is not mathematically estimated, but is empirically constructed on the basis of all of the characteristics of the original data distribution, including the ones generally considered to be contaminating factors (e.g., skewness, ceiling effect, heavy tails, outliers, etc.). This makes bootstrap estimations more adequate when the data do not follow a normal probability distribution.

Bootstrap sampling is also useful for describing the sampling distribution of estimators for which the sampling properties are unknown or unavailable. For example, bootstrap has been used to estimate the variability of eigenvalues and the stability of factor loadings (Chatterjee, 1984; Scott, Thompson, \& Sexton, 1989; Thompson, 1988), measure the stability of item bias index in test construction (Harris \& Kolen, 1989), and measure the sampling variability of various estimators in multiple- 
regression equations (Efron \& Gong, 1983; Freedman, 1981; Lunnenborg, 1987; Stine, 1989).

Although bootstrap simulation generally involves redrawn samples that are the same size as the original sample, it is also possible to vary the size of the bootstrap samples to compare various estimator distributions obtained for different sample sizes. This innovative use of bootstrap can prove useful in planning research. First, it allows one to choose between various sample sizes given some desired precision level and, second, bootstrap techniques can be used for power estimation. An approach commonly used for power analysis is to estimate effect size from data that are already available. Then, using the proper table (e.g., Cohen, 1977; Kraemer \& Theimann, 1987), one can get an indication of the statistical power of the design at a given alpha level and, thus, choose an appropriate sample size. The main problem with this approach to power estimation is that the power tables are based on the assumption of normal population distributions. The bootstrap approach to power estimation does not require any distribution assumptions and makes the estimation a straightforward process. While using bootstrap, the proportion of resampling samples leading to a statistically significant estimator at a given alpha level is computed and used as a power estimate. By varying the bootstrap sample size or the significance level, or by using alternative estimators, the power of various research designs can be compared.

To illustrate this application, we used SIMSTAT to estimate the power of a planned evaluation study designed to assess the effect of a community follow-up program on alcohol-consumption patterns in homeless men, as indicated by the number of intoxicating substances used. The data we used were obtained from a pilot study involving 29 subjects (Péladeau, 1988). We compared the estimated statistical power associated with two samples with 20 subjects and 60 subjects, respectively. We also compared $t$ tests and Mann-Whitney $U$ tests to determine which one had the greater chance of yielding significant results. To do so, four bootstrap simulations were performed, using two different bootstrap sample sizes and two distinct comparative analyses. For each simulation, the proportion of samples yielding a significant result at three alpha levels was computed. Summarized in Table 1, the results show, for $\alpha=.05$ (two-tailed), a very slight advantage for the Mann-Whitney $U$ over the $t$ test with a sample size of 20; the former test was found to be significant for $37.9 \%$ of the bootstrap samples, compared with $34.5 \%$ using the $t$ test. ${ }^{2}$ The simulation with sample sizes of 60 increased the power of the design to $84.5 \%$ for the Mann-Whitney $U$ test, compared with $78.7 \%$ for the $t$ test.

This approach to power estimation thus has the additional advantages of supplying an empirical basis for choosing between two different statistical strategies. Although the current program is limited in the number of
Table 1

Proportions of Significant Bootstrap Samples at Three Alpha Levels, Two-Tailed, for $t$ Test and Mann-Whitney $U$

\begin{tabular}{|c|c|c|}
\hline \multirow[b]{2}{*}{ Alpha Levels } & \multicolumn{2}{|c|}{ Sample Size } \\
\hline & 20 & 60 \\
\hline \multicolumn{3}{|c|}{$t$ Test } \\
\hline$\alpha=.01$ & 16.6 & 55.8 \\
\hline$\alpha=.05$ & 34.5 & 78.7 \\
\hline$\alpha=.10$ & 46.2 & 86.5 \\
\hline \multicolumn{3}{|c|}{ Mann-Whitney $U$ Test } \\
\hline$\alpha=.01$ & 15.3 & 65.5 \\
\hline$\alpha=.05$ & 37.9 & 84.5 \\
\hline$\alpha=.10$ & 51.1 & 91.0 \\
\hline
\end{tabular}

statistical tests that can be studied, the bootstrap technique could, theoretically, be used to compute power estimates for estimators for which power tables are not yet available.

Varying the bootstrap sample size could be a convenient tool in teaching statistics. Because it allows one to observe the effects of varying sample size on sample estimator distributions and statistical power, bootstrap simulation could be used to illustrate concepts such as sampling theory or central limit theorem (see also Bradley, 1989, for a discussion on the pedagogical use of Monte Carlo simulations). Additionally, bootstrap might be helpful in studying the effect that violation of the normality assumption would have on some estimators. In this respect, the main advantage of bootstrap simulation over other Monte Carlo methods is that it provides a direct assessment of sampling distribution from real-world data provided by the user, rather than from data generated by assumed mathematical functions.

Although there is little bootstrap software available today, resampling is gaining popularity among behavioral and social scientists. Programs such as those developed by Lunnenborg (1987) and Van Bergem, Ditrichs, and Simon, (1986) offer bootstrap techniques. However, these programs are mainly designed for specific statistical analysis. SIMSTAT was designed to be a general-purpose tool for performing bootstrap estimation of several descriptive, uni- and bivariate statistics. In addition, it offers the possibility of varying the bootstrap sample size. The program is easy to use and supports several popular file formats.

\section{Program Description}

SIMSTAT is a statistical program that was developed to supply the social scientist with bootstrap techniques for uni- and bivariate analysis. The program uses pull-down menus and context-sensitive help.

Bootstrap analysis. The program implements bootstrap analysis and allows an examination of sampling distributions for seven descriptive estimators of a single variable (i.e., mean, variance, standard deviation, standard error, median, skewness, kurtosis) and of the following 20 es- 
timators involving two variables: Kendall's tau a and b; Kendall-Stuart's tau c; symmetric and asymmetric Somers's $D$; Goodman-Kruskal's gamma; Student's $t$ and $F$; Pearson's $r$; Spearman's rho; regression slope and intercept; Mann-Whitney's $U$; Wilcoxon's $W$; differences between means as well as variances; sign test; Kruskal-Wallis's one-way ANOVA by rank; and median test.

The output provides information that includes descriptive statistics, frequency tables, histograms, and percentile tables. The program also computes bootstrap confidence intervals (nonparametric and bias corrected) and displays power estimates for up to four alpha levels. The default sample size for bootstrap is the original sample size, although the user might specify any size between 10 and 20,000 .

SIMSTAT also provides another variation of bootstrap which, in contrast to standard bootstrap sampling, is done on independent observations. In the law school example, the data for both variables were drawn from the same observation unit (i.e., bivariate sampling). For the alternate method, the data are drawn on each variable independently. The overall correlation that would result from such a simulation should theoretically be near zero. Assuming the null hypothesis, this allows one to estimate Type I error. The program also allows one to perform various statistical analyses such as frequency analysis, crosstabulation, and multiple regression on each bootstrap sample.

Basic analysis. SIMSTAT is also a comprehensive statistical package that provides many descriptive statistics (mean, variance, standard deviation, skewness, kurtosis, etc.) and frequency analysis (frequency table, barchart, histogram, percentile tables). SIMSTAT provides twoway cross-tabulation with nominal (chi, phi, and Cramer's $V$ ), ordinal (Kendall's tau b and tau c, Somers's $D, D_{x y}$, $D_{y x}$, Goodman-Kruskal's gamma, lambda, and contingency coefficient) and interrater statistics (percentages of agreement, Cohen's kappa, etc.). The program also computes paired and independent $t$ tests, linear, nonlinear, and multiple regression, and Pearson's correlation including covariance and cross-product deviation estimates. SIMSTAT also computes nonparametric statistics, including MannWhitney's $U$ test, Wilcoxon's signed ranks test, sign test, Kruskal-Wallis's ANOVA, two-sample KolmogorovSmirnov, Moses's test of extreme reaction, median test for two or more samples, and association matrix for nonparametric estimators (Spearman's $r$, Somers's $D, D_{x y}$, and $D_{y x}$, Goodman-Kruskal's gamma, Kendall's tau a and tau b, and Kendall-Stuart's tau c).

Data input. SIMSTAT handles up to 500 variables and 30,000 cases; simulation procedures allow between 10 and 20,000 resamplings. These limits can be lower, depending on the amount of computer memory available. The program handles four file types for data input: SPSS/PC + (compressed and uncompressed), dBase III and IV, Lotus $1-2-3$, and ASCII. Alternatively, data can be entered from the keyboard.

Output control. The output generated is either displayed on screen, saved on disk, and/or directed to the printer. The program supports black and white or color monitors and can display text using 25,43 , or 50 lines on EGA and VGA monitors. The program also provides support for some printer configuration.

Hardware requirements. SIMSTAT runs on any IBM $\mathrm{PC} / \mathrm{XT}, \mathrm{AT}, \mathrm{PS} / 2$, or compatible with at least $400 \mathrm{~K}$ free RAM and requires MS-DOS or PC-DOS V. 2.0 or higher. A math coprocessor is not needed but can be used if available; it is recommended for extensive bootstrap simulations on large samples.

\section{Availability}

SIMSTAT is distributed as a shareware program. A nonregistered version of the program can be obtained from major bulletin boards, from shareware distributors, or by writing to Normand Péladeau, 5000 Adam Street, Montreal, QC, Canada HIV IW5.

Please include a \$5 U.S. check or money order to cover media and mailing costs. A registered version of the program can also be obtained from the author for $\$ 55$ U.S. Registration benefits include a printed manual, technical support, and a free update.

\section{REFERENCES}

Bradley, D. R. (1989). Computer simulation with DATASIM. Behavior Research Methods, Instruments, \& Computers, 21, 99-112.

Chatterjee, S. (1984). Variance estimation in factor analysis: An application of the bootstrap. British Journal of Mathematical \& Statistical Psychology, 37, 252-262.

COHEN, J. (1977). Statistical power analysis for the behavioral sciences (2nd ed.). New York: Academic Press.

Diaconis, P., \& EFron, B. (1983). Computer-intensive methods in statistics. Scientific American, 248(5), 116-130.

Efron, B. (1979). Computers and the theory of statistics: Thinking the unthinkable. SIAM Review, 21, 460-480.

EFRON, B. (1981). Nonparametric estimates of standard error: The jackknife, the bootstrap, and other resampling methods. Biometrika, 68 , 589-599.

Efron, B., \& Gong, G. (1983). A leisurely look at the bootstrap, the jackknife and cross-validation. American Statistician, 37, 36-48.

FreEDMAN, D. A. (1981). Bootstrapping regression models. Annals of Statistics, 9, 1218-1228.

Harris, D. J., \& Kolen, M. J. (1989). Examining the stability of Angoff's Delta item bias statistic using the bootstrap. Educational \& Psychological Measurement, 49, 81-87.

Kraemer, H. C., \& Thiemann, S. (1987). How many subjects? Statistical power analysis in research. Beverly Hills, CA: Sage.

LUNNENBORG, C. E. (1987). Bootstrap applications for the behavioral sciences. Educational \& Psychological Measurement, 47, 627-629.

MiCCERI, T. (1989). The unicorn, the normal curve, and other improbable creatures. Psychological Bulletin, 155, 155-166.

Péladeau, N. (1988). Analyse d'impact d'un programme de suivi communautaire. Montreal: Centre d'Accueil Préfontaine.

Scott, R. L., Thompson, B., \& Sexton, D. (1989). Structure of a short form of the questionnaire on resources and stress: A bootstrap factor analysis. Educational \& Psychological Measurement, 49, 409-419.

STINE, R. (1989). An introduction to bootstrap methods. Sociological Methods \& Research, 18, 243-289.

Thompson, B. (1988). Program factrap: A program that computes bootstrap estimates of factor structure. Educational \& Psychological Measurement, 48, 681-686.

Van Bergem, P., Ditrichs, R., \& Simon, S. (1986). A BASIC program for nonparametric analyses of proximity matrices. Behavior $R e$ search Methods, Instruments, \& Computers, 18, 407-408. 


\section{NOTES}

1. Actually, the bootstrap method does not make an estimator more robust. However, it provides a more accurate estimate of its sampling variability and can thus provide some information on its robustness (Stine, 1989).

2. To make sure that the difference between the estimators cannot be attributed to differences in bootstrap samples, the same random sequence was used for both estimators.

APPENDIX A

Average Scores on the Undergraduate Grade Point Average (GPA) and the Law School Admission Test (LSAT)

\begin{tabular}{cc}
\hline GPA & LSAT \\
\hline 3.39 & 576 \\
3.30 & 635 \\
2.81 & 558 \\
3.03 & 578
\end{tabular}

APPENDIX A (continued)

\begin{tabular}{cc}
\hline GPA & LSAT \\
\hline 3.44 & 666 \\
3.07 & 580 \\
3.00 & 555 \\
3.43 & 661 \\
3.36 & 651 \\
3.13 & 605 \\
3.12 & 653 \\
2.74 & 575 \\
2.76 & 545 \\
2.88 & 572 \\
2.96 & 594 \\
\hline
\end{tabular}

Note-From "Computers and the theory of statistics: Thinking the unthinkable" by B. Efron, 1979, SIAM Review, 21, p. 462. Copyright 1979 by the Society for Industrial and Applied Mathematics, Philadelphia, PA. Reprinted by permission.

\section{APPENDIX B}

Sample Bootstrap Simulation on the Law School Data

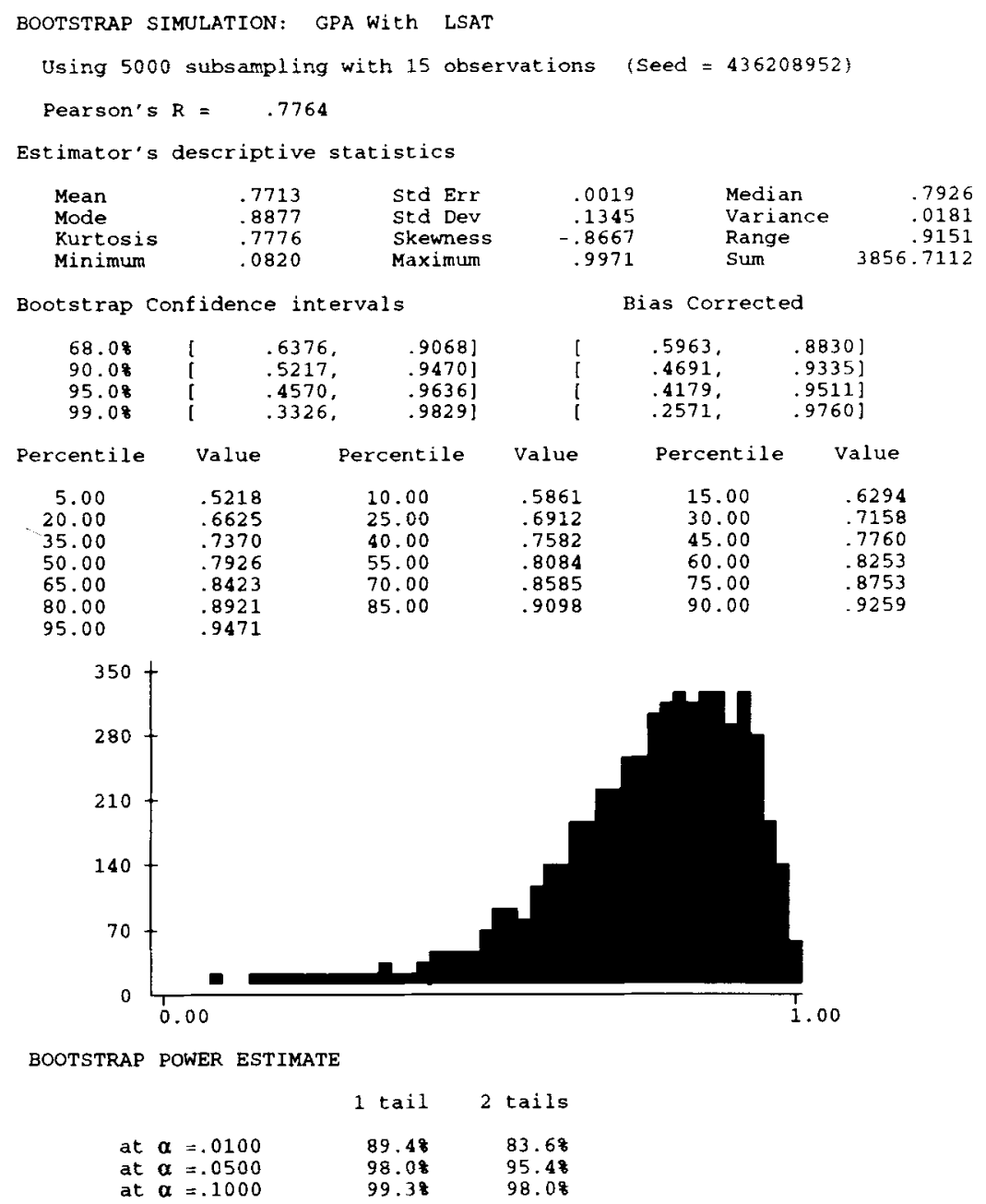

(Manuscript received July 10, 1991;

revision accepted for publication January 25, 1993.) 